\title{
Recurrent myoepithelial carcinoma of the submandibular gland treated by rAd-p53 combined with radiotherapy: A case report
}

\author{
LIN YANG $^{1}$, XIANGXUN CHEN ${ }^{1}$, HONGYAN WANG ${ }^{1}$ and QINGMING SHI ${ }^{2}$ \\ ${ }^{1}$ Department of Radiation Oncology, The First Affiliated Hospital of Anhui Medical University; ${ }^{2}$ Department of Oncology, \\ Anhui Chest Hospital of Anhui Medical University, Hefei, Anhui 230022, P.R. China
}

Received March 13, 2015; Accepted April 1, 2016

DOI: $10.3892 / \mathrm{ol} .2016 .4746$

\begin{abstract}
The aim of the present study was to report the case of a patient with recurrent myoepithelial carcinoma of the submandibular gland without progression for five years following treatment. A 71-year-old male patient presented to hospital with a painless swelling in the region of the right submandibular gland, and received a radical neck dissection on January 29,2008 . A nodule of $\sim 7 \times 4 \times 2 \mathrm{~cm}$ was identified at the site of the right submandibular gland, and the pathological results revealed a diagnosis of myoepithelial carcinoma of the right submandibular gland with no lymph node metastasis. However, this case developed local recurrence with wide-spread metastasis in the lungs. Between April and October 2008, the patient underwent several treatment regimens and demonstrated no improvement following 6 cycles of chemotherapy. From then on, the patient was treated with recombinant adenoviral-p53 (rAd-p53) combined with radiotherapy using a 6 millivolt medical linear accelerator. The foci were relieved and the cancer demonstrated no signs of progression during the 5-year follow-up. rAd-p53 combined with radiotherapy was useful for treating myoepithelial carcinoma of the submandibular gland.
\end{abstract}

\section{Introduction}

Myoepithelial carcinoma, or malignant myoepithelioma, is a malignant carcinoma that affects the salivary gland. Epithelial-myoepithelial carcinoma was first classified by the World Health Organization (WHO) as a rare, low grade of malignancy tumor, which accounts for $\sim 1 \%$ of all primary tumors of salivary glands (1). The tumor is generally composed of variable proportions of two cell types: An inner layer of duct lining cells and an outer layer of clear cells, which typically

Correspondence to: Professor Qingming Shi, Department of Oncology, Anhui Chest Hospital of Anhui Medical University, 397 Jixi Road, Hefei, Anhui 230022, P.R. China

E-mail: qingmingshicn@126.com

Key words: recombinant adenoviral-p53, malignant myoepithelial, carcinoma, radiotherapy form double-layered duct-like structures. Clear cells, which are of myoepithelial origin, often predominate in number. There is a female predominance, with a peak occurrence in the seventh decade (2). Myoepithelial carcinoma commonly recurs locally after resection, while distant metastasis rarely occurs (3). At present, few studies report the effectiveness of introduced treatment regimes; in addition, there is an evident lack of understanding of the biological characteristics of myoepithelial carcinoma and experience of treatment. The present study reports the case of patient with recurrent myoepithelial carcinoma with no cancer progression for five years following treatment.

\section{Case report}

A 71-year-old male patient presented to the Department of Oncology, The First Affiliated Hospital of Anhui Medical University (Hefei, Anhui, China) in January 2008 with swelling in the region of the right submandibular gland. On January 29, 2008, the patient received a neck computed tomography (CT) scan, the result of which revealed a swelling of approximately $7 \times 4 \times 2 \mathrm{~cm}$ in size, with right lymph node swelling. The swelling was subsequently removed as a I-field lymphadenectomy was performed. Following the procedure, a distinct clinicopathological entity of salivary gland tumors and a morphological structure similar to clear cell tumor was found, and the pathological results confirmed the diagnosis of myoepithelial carcinoma of the right submandibular gland, with no lymph node metastasis. However, in April 2008, the patient presented to the hospital with swelling in the same region with deradenoncus on the right side. A chest X-ray showed that certain foci of unequal sizes were present in the lungs and presented wide-spread metastasis (Fig. 1A). A chest CT scan showed wide-spread metastasis in the lungs (Fig. 1B). In June 2008, CT scan indicated that the swelling in the region of the right submandibular gland was enlarged with deradenoncus on the right side (Fig. 1C). A physical examination indicated that the swelling boundary in the region of the right submandibular gland was obscure, hard and could be touched, and the swelling was approximately $6 \times 5 \mathrm{~cm}$ in size. Furthermore, numerous swollen lymph nodes were palpable in the region of the right submandibular gland, the maximum diameter of which was $3 \mathrm{~cm}$ and the boundary was not clear. 


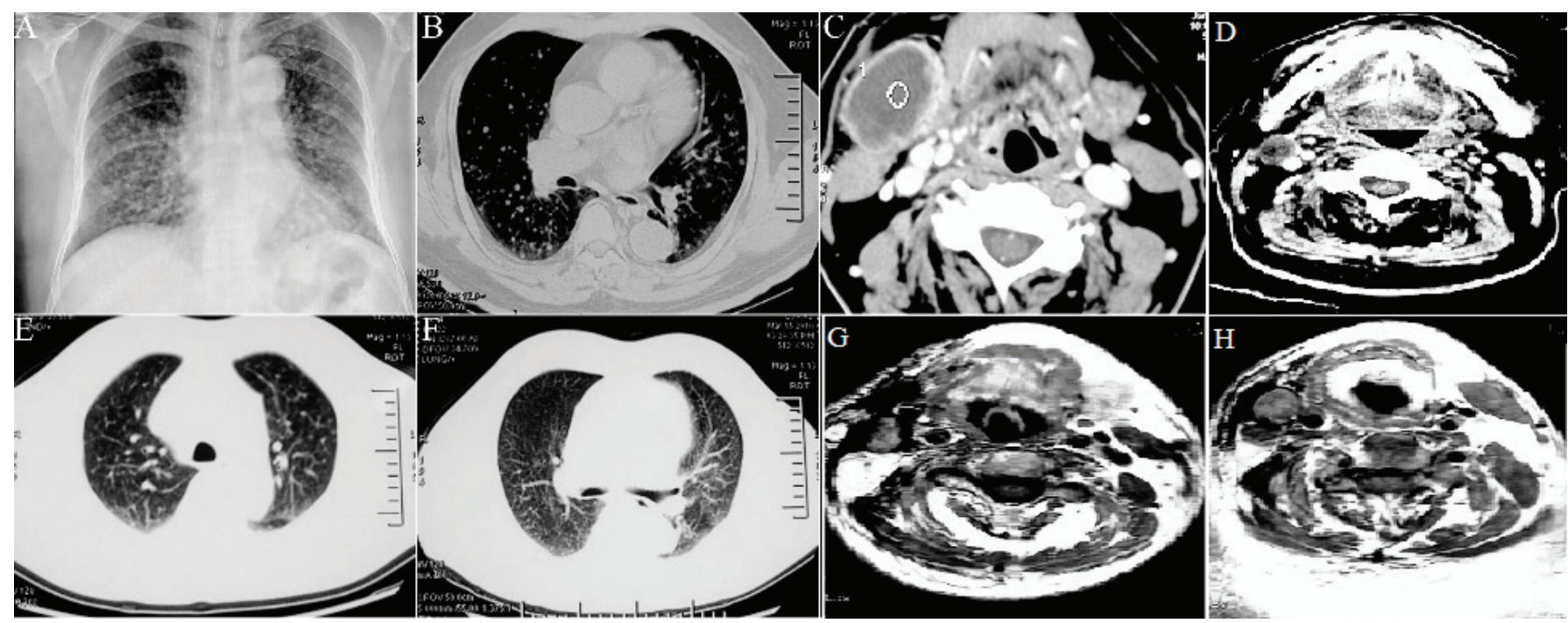

Figure 1. Changes in the lung, cervical lymph node metastasis and primary tumor lesions prior to and subsequent to treatment. (A) In April 2008, a chest X-ray showed nodular shadows of unequal sizes and densities in the lungs, which indicated hematogenous metastasis carcinoma in the lungs. (B) In April 2008, a chest CT scan showed nodular shadows of unequal sizes and densities in the lungs. (C) In June 2008, an MRI scan showed that the swelling in the region of the right submandibular with deradenoncus. (D) In August 2009, an MRI scan showed the swelling of right submandibular gland and neck lymph node were evidently reduced. (E) In December 2009, the miliary foci were evidently reduced on CT. (F) In March 2010, a chest CT scan showed that the foci had disappeared in the lungs. (G and H) In November 2011, magnetic resonance imaging scans showed that the primary recurrent foci was in remission and that the residual region demonstrated partial fiber change. CT, computed tomography; MRI, magnetic resonance imaging.

Between April 2008 and October 2008, the patient was administered 2 cycles of a cisplatin + fluorouracil chemotherapy regimen $\left[70 \mathrm{mg} / \mathrm{m}^{2}\right.$ intravenous (iv) cisplatin on day 1 and $750 \mathrm{mg} / \mathrm{m}^{2} /$ day iv fluorouracil on days $1-5$, every 28 days] and 4 cycles of a cisplatin + doxorubicin + cyclophosphamide regimen $\left(70 \mathrm{mg} / \mathrm{m}^{2}\right.$ iv cisplatin on day $1,40 \mathrm{mg} / \mathrm{m}^{2}$ iv doxorubicin on day 1 and $70 \mathrm{mg} / \mathrm{m}^{2}$ cyclophosphamide on day 1 , every 21 days) in the Department of Oncology, Anhui Chest Hospital of Anhui Medical University, Hefei, China. Due to disease progression, the patient was diagnosed with recurrent myoepithelial carcinoma of the submandibular gland in October 2008 and transferred to Department of Radiation Oncology, The First Affiliated Hospital of Anhui Medical University, Hefei, China. Subsequently, the patient received radiotherapy with a 6-MV medical linear accelerator (Varian Medical Systems, Inc., Palo Alto, CA, USA) [test dose, (DT) $70 \mathrm{~Gy} / 35$ fractions] for the swelling in the right neck. However, the effects of the treatment were not evident, so the patient was administered a multi-target points injection supplemented with recombinant adenoviral-p53 (rAd-p53; catalog no. 20080401; SiBono GenTech Co., Ltd., Shenzhen, China) for the tumor, once per week for two weeks, combined with radiotherapy (DT, $10 \mathrm{~Gy} / 5$ fractions) at intervals.

In August 2009, six months subsequent to the treatment, a CT scan revealed that the swelling of the right submandibular gland and lymph nodes were evidently smaller (Fig. 1D). In addition, the lungs were not treated specifically, and the patient only received 2.8-g spirulina tablets orally, three times per day and $10 \mathrm{~g}$ Qing Fei San Jie Wan (a herbal remedy), three times per day. In December 2009, the miliary foci were evidently reduced in size and number (Fig. 1E). A chest $\mathrm{CT}$ revealed that the foci had been fully removed in March 2010 (Fig. 1F). A magnetic resonance imaging (MRI) scan in November 2011 revealed that the primary recurrent foci was in remission, and that the residual region demonstrated a partial fiber change (Fig. 1G and H). Over a follow-up of 5 years (6-month intervals), on CT and MRI, the patient did not present with local recurrence, the swelling in the neck lymph node had disappeared and the primary swelling demonstrated a partial fiber change. Furthermore, the intrapulmonoary nodule was in remission and the condition of the patient returned to normal, without a cough, chest distress or short breath. The present study was conducted in accordance with the Declaration of Helsinki and with approval from the Ethics Committee of Anhui Medical University, Hefei, China. Written informed consent was obtained from the patient.

\section{Discussion}

In recent years, myoepithelial carcinoma has been shown to be a moderately- to poorly-differentiated malignant tumor that presents with high grade malignancy and strong invasion, and the majority of patients are expected to have a poor prognosis (4-6). Myoepithelial cells are located in the glands. The cells contact and expel the secretions of the exocrine glands, including the salivary, sweat, bartholin, prostate and lacrimal glands, the galactophores and pancreas, and the tissue originates from ectoderm and has epithelial features $(7,8)$. Epithelial-myoepithelial carcinomas of the salivary gland can develop malignancy from benign myoepithelial carcinomas, with the exception of primary carcinomas $(4,5)$.

It has previously been indicated that epithelial myoepithelial carcinomas belong to a category of low grade malignant carcinomas and have a relatively good prognosis; however, recurrence is often extremely frequent due to the invasive growth pattern, which was indicated in an analysis of four cases of epithelial-myoepithelial carcinoma of the salivary gland (5). However, one study also followed up one patient for 3 years, who showed no recurrence or metastasis (9). Yu et al (6) examined 19 cases of epithelial-myoepithelial carcinoma of 
the salivary gland and identified 13 cases that experienced recurrence numerous times. Overall, 9 cases succumbed to the tumor. In addition, Yu et al (6) identified certain clinical features of the carcinoma, including: Arising most frequently within the parotid glands, followed by the submandibular glands; rapidly enlarging mass with extensive invasion of the surrounding tissues in certain cases; low rate of cervical lymph node metastasis but high rate of distant metastasis; extremely frequent recurrence following surgical excision; and poor prognosis. Furthermore, an evident difference in DNA levels between benign carcinomas and malignant myoepithelial carcinomas was indicated, as the myoepithelial carcinoma cells presented varied biological characteristics. In 2014, a study reported that myoepitheliomas occur in soft tissues, and detailed clinical features, radiographic examinations and histological and immunohistochemical characteristics all revealed that myoepithelial soft tissue sarcoma was a special type of myoepithelial malignancies (10). Another study indicated that, as a multi-cell hybrid myoepithelioma, the cytoplasmic mucin content was the important feature of myoepithelioma (11). Cros et al (12) studied the treatment-associated gene mutations and target expressions of 123 patients with malignant salivary gland tumors, and revealed that 107 patients had varied gene mutations and target expressions, which provided the basis for additional molecular targeted therapy.

The treatment process of the present study also indicated that this particular myoepithelial carcinoma had high rate of distant metastasis and frequent recurrence, which supported the findings of the previous study by $\mathrm{Yu}$ et al (6). Although the tumor was reduced in size, the expected effect was not reached following he administration of DT $70 \mathrm{~Gy} / 35$ fractions of radiotherapy; however, the tumor size was evidently following the administration of the rAd-p53 injection in the carcinoma and the increased dose of radiotherapy. The results of the treatment may be due to several reasons. For example, the carcinoma size was large and had numerous anoxic cells that were resistant to radiotherapy; however, when rAd-p53 was injected into tumor it may have suppressed the growth of tumor and induced tumor cells apoptosis, even inhibited tumor tissue revascularization, which may have decreased the number of anoxic cells and allowed the radiation to exert its effect. Notably, during the 5-year follow-up, the patient did not present with local recurrence. This finding suggests that patients receiving surgery may be able to undergo radiotherapy for the prevention of recurrence, based on the treatment effects of rAd-p53 combined with radiotherapy on the tumor in the present study.

The present study reports a case of recurrent myoepithelial carcinoma of the submandibular gland, which was relieved following treatment with $\mathrm{Ad}-\mathrm{p} 53$ combined with radiotherapy and was without progression for 5 years.

\section{References}

1. Politi M, Robiony M, Avellini C and Orsaria M: Epithelial-myoepithelial carcinoma of the parotid gland: Clinicopathological aspect, diagnosis and surgical consideration. Ann Maxillofac Surg 4: 99-102, 2014.

2. Seifert $\mathrm{G}$ and Sobin LH: Epithelial-myoepithelial carcinoma. In: World Health Organization International Histological Classification of Tumours. Histological Typing of Salivary Gland Tumours. 2nd edition. Springer-Velag, Berlin, pp23-24, 1991.

3. Donath K, Seifert G and Schmitz R: The diagnosis and ultrastructure of tubular salivary duct carcinoma. Virchows Arch 356: 16-31, 1972 (In German).

4. Lima FJ, Porto DE, Cavalcante JR, Oka SC and Godoy GP: Epithelial-myoepithelial carcinoma of high grade transformation: The case report in the buccal mucosa. Open Dent J 6: 111-117, 2012.

5. Li Y, Kim HS, Kang MS, Shin SH, Koo KH, Kim CM, Kim KH, Peck C, Bae HI, Jeong JY, et al: A spontaneous epithelial-myoepithelial carcinoma of the submandibular gland in a sprague-dawley rat. J Toxicol Pathol 26: 67-72, 2013.

6. Yu G, Ma D and Sun K: The behaviour and treatment of myoepithelial carcinoma of salivary glands. Zhonghua Kou Qiang Yi Xue Za Zhi 32: 67-69, 1997 (In Chinese)

7. Lee JW, Myung NH and Suh MW: Epithelial-myoepithelial carcinoma of external auditory canal evolving from pleomorphic adenoma. Korean J Audiol 16: 148-151, 2012.

8. Arora SK, Sharma N and Bhardwaj M: Epithelial myoepithelial carcinoma of head and neck region. Indian J Otolaryngol Head Neck Surg 65 (Suppl 1): S163-S166, 2013.

9. Aydin S, Taskin U, Ozdamar K, Yücebas K, Sar M, Oktay MF and Tetikkurt US: Case study of a parotid gland adenocarcinoma dedifferentiated from epithelial-myoepithelial carcinoma. Case Rep Otolaryngol 2014: 629054, 2014.

10. Mahdi Y, Zouaidia F, Zouhair A, Azouz M, Znati K, Jahid A, Berrada MS, Bernoussi Z, Mansouri F, El Yaacoubi M and Mahassini N: Combined myoepithelial carcinoma and myoepithelioma in soft tissue: A case report and review of the literature. J Med Case Rep 8: 317, 2014.

11. Gnepp DR: Mucinous myoepithelioma, a recently described new myoepithelioma variant. Head Neck Pathol 7 (Suppl 1): S85-S89, 2013.

12. Cros J, Sbidian E, Hans S, Roussel H, Scotte F, Tartour E, Brasnu D, Laurent-Puig P, Bruneval P, Blons $\mathrm{H}$ and Badoual C: Expression and mutational status of treatment-relevant targets and key oncogenes in 123 malignant salivary gland tumours. Ann Oncol 24: 2624-2629, 2013. 